\title{
Self-assembly of Fine Particles on Patterned Wettability in Dip Coating and Its Scale Extension with Contact Printing
}

\author{
Yoshihito KANAMORI ${ }^{1}$, Arata KANEKO ${ }^{2}$, Nobuyuki MORONUKI ${ }^{3}$, Takafumi KUBO ${ }^{4}$ \\ 1Tokyo Metropolitan University, Japan, kanamori-yoshihito@sd.tmu.ac.jp \\ 2Tokyo Metropolitan University, Japan, kaneko-arata@tmu.ac.jp \\ ${ }^{3}$ Tokyo Metropolitan University, Japan, moronuki@tmu.ac.jp \\ ${ }^{4}$ Tokyo Metropolitan University, Japan, kubo-takafumi@ed.tmu.ac.jp
}

\begin{abstract}
:
This study demonstrates the application of dip coating to self-assembly of fine particles on a substrate that is covered with line-and-space pattern of hydrophilic $\left(\mathrm{SiO}_{2}\right)$ and hydrophobic materials (OTS). The pattern was fabricated on a substrate by lithography first. The substrates were drawn up from the water-based suspension in which particles were dispersed and the particles self-assembled on the hydrophilic region only forming the packed-structure. Then, contact printing was applied for the patterning to extend the scale. The scale was extended up to $10 \mathrm{~mm} \times 10 \mathrm{~mm}$ while keeping the self-assemble performance.
\end{abstract}

Keyword: Particle, Self-assembly, Wettability, Dip coating, Contact printing

\section{Introduction}

Assembly of micro/nano-particles allows new functional devices such as microlens [1], biosensor [2-3], etching mask [4], and photonic crystal [5-8]. So far, various assembly technique were tried, e.g., laser trapping [9], SPM manipulation [10], and dip coating [11-13]. However, any assembly technique that compromises various requirements such as accuracy, productivity, and spatial selectivity has not been established. It is well known that dip coating process, drawing-up a substrate from suspension in which particles are dispersed, enables the self-assemble of particles on a hydrophilic substrate [11-13]. The particles autonomously pack in hexagonal due to the capillary forces among them as the evaporation of liquid in suspension. This method can provide an easier way and higher productivity to assemble particles comparing with the other technique, but it is difficult to assemble particles selectively on specific position.

It has been reported that pure water selectively wet and/or spread on the specially patterned substrate with an array of hydrophilic and hydrophobic materials [14]. Dip coating with hydrophilic/hydrophobic pattern can locate particles on desired region with high productivity. The hydrophilic/hydrophobic pattern was fabricated by Electron beam (EB) lithography method and could be assembled particle on this pattern [14]. But the pattern size is limited because the time required for lithography is proportional to the size. Therefore, it is difficult to assemble particle on large area.

Contact printing technique has been applied for various patterning [15-21]. This process is effective especially in the case of large scale patterning because the patterning can be carried out in parallel over the mold size. However, it is not necessarily applied for hydrophilic/hydrophobic patterning and optimum conditions have not been made clear.

This paper firstly demonstrates the self-assembly of fine particles by dip coating using the hydrophilic/hydrophobic patterned substrate prepared with EB-lithography. Then, the contact printing technique is applied including the process optimizing intending the scale extension of the pattern.

\section{Self-assembly mechanism}

Figure 1 shows the schematic illustration of dip coating with hydrophilic/hydrophobic pattern [14]. Patterned substrate is dipped into the suspension in which particles are dispersed and then draw up at constant speed $V$ and angle $\varphi$. The suspension locally spreads on hydrophilic

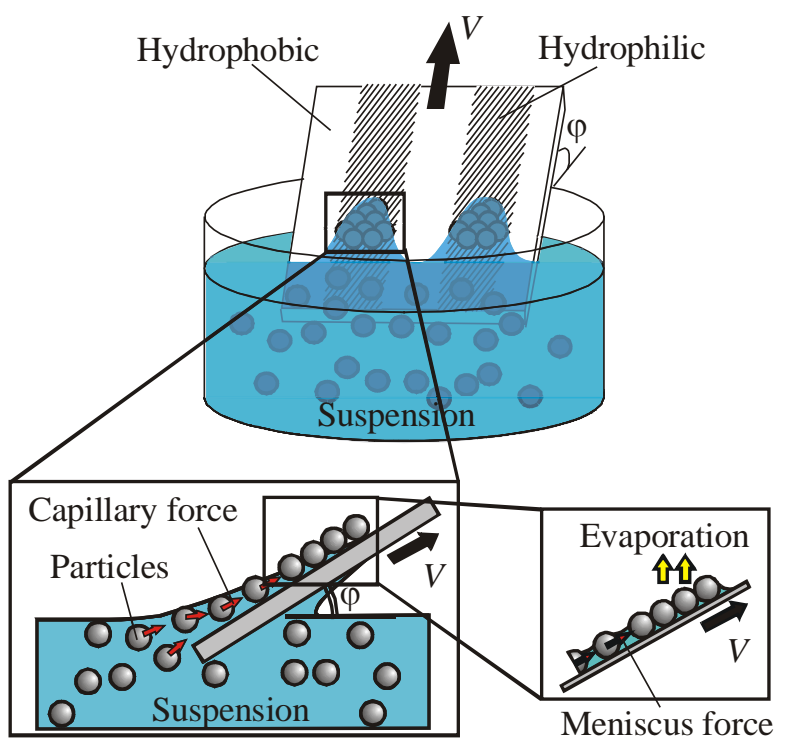

Figure 1: Self-assembled process on hydrophilic/hydrophobic-pattern. 
region with the help of capillary force. According with the evaporation of solution particles self-assembled forming hexagonally packed-structure due to the meniscus forces among them.

\section{Demonstration of self-assembly to the substrate fabricated by EB-lithography}

\subsection{Preparation of the substrates}

Figure 2 shows the process steps of EB-lithography patterning [14]. Firstly, Si (100) wafer was soaked in $\mathrm{H}_{2} \mathrm{SO}_{4}: \mathrm{H}_{2} \mathrm{O}_{2}=3: 1$ solution kept at 70 degrees for 2 hours to form thin $\mathrm{SiO}_{2}$ layer. Then, the octadecyltrichloro -silane (OTS) self-assembled monolayer (OTS-SAM) was deposited by dipping in OTS-toluene solution (1 wt.\%) for 2h, and the substrate was soaked in $\mathrm{H}_{2} \mathrm{SO}_{4}$ to clean up excess OTS. OTS reacts well with hydroxyl groups, including the environmental water vapor. Therefore, this process was carried out in a glove box purged with dry $\mathrm{N}_{2}$ gas. Then, electron beams were irradiated to remove OTS with specified pattern. The dose amount was set at 4000 $\mu \mathrm{C} / \mathrm{cm}^{2}$. Finally, Irradiated OTS was removed and $\mathrm{SiO}_{2}$ layer was produced in $\mathrm{H}_{2} \mathrm{SO}_{4}-\mathrm{H}_{2} \mathrm{O}_{2}$ solution.

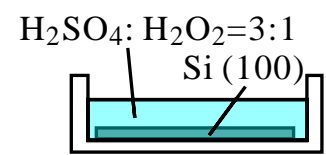

(1) Oxidization

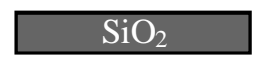

(2) $\mathrm{SiO}_{2}$ layer

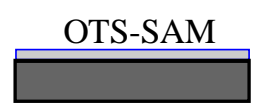

(3) Deposition of OTS

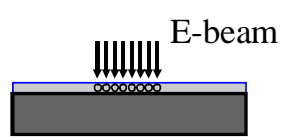

(4) Dose on OTS
Figure 2: Process step for EB-lithography.

Figure 3 shows the atomic force microscope (AFM) image and contact angle that was measured on wider samples. Step height between OTS and $\mathrm{SiO}_{2}$ was $1.4 \mathrm{~nm}$, which corresponded to OTS molecular length and thus this layer is confirmed as monolayer OTS (OTS-SAM). The contact angle of $\mathrm{SiO}_{2}$ region $\theta_{\mathrm{SiO}}$ was 34 and that of OTS region $\theta_{\text {OTS was }} 108$ degrees respectively for pure water of $1 \mu$ l. The OTS region had enough hydrophobic property because the contact angle was larger than 90 degrees. However, the $\mathrm{SiO}_{2}$ region had weak hydrophilic property because the contact angle was rather large. Ideally, the contact angle of $\mathrm{SiO}_{2}$ is less than 10 degrees. These properties may affect the self-assembly process.

The patterned area was $1 \mathrm{~mm} \times 1 \mathrm{~mm}$, and it took for 2 hours. EB-lithography is convenient for small lot fabrication because no glass mask is necessary, but, it is difficult to adopt large scale fabrication because it takes long time.

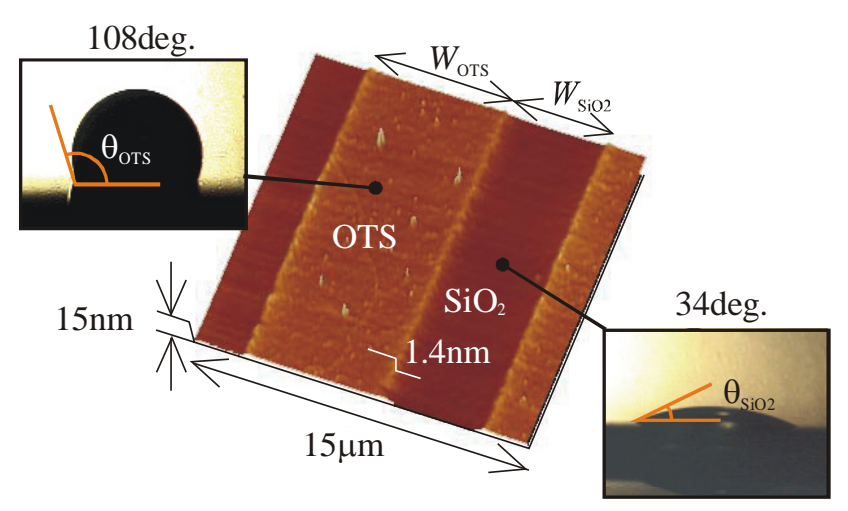

Figure 3: AFM image and contact angle on the pattern Fabricated by EB-lithography.

\subsection{Setup for dip-coating}

Figure 4 shows the schematic illustration of the experimental setup. The system consists of an inchworm actuator, a rotation stage, and a reservoir. The inchworm-type linear stage draw up a substrate at required drawing speed $V$. The rotational stage was used to set the drawing angle $\varphi$ ranging from 0 to 90 degrees. The reservoir was filled with a suspension in which polystyrene particles of $1 \mu \mathrm{m}$ in diameter disperses in pure water at $1 \%$ concentration. The reservoir size was $\phi 20 \mathrm{x}$ $5 \mathrm{~mm}$. This system is set in a clean room in which temperature and humidity are kept at 20 centigrade and $50 \%$ respectively.

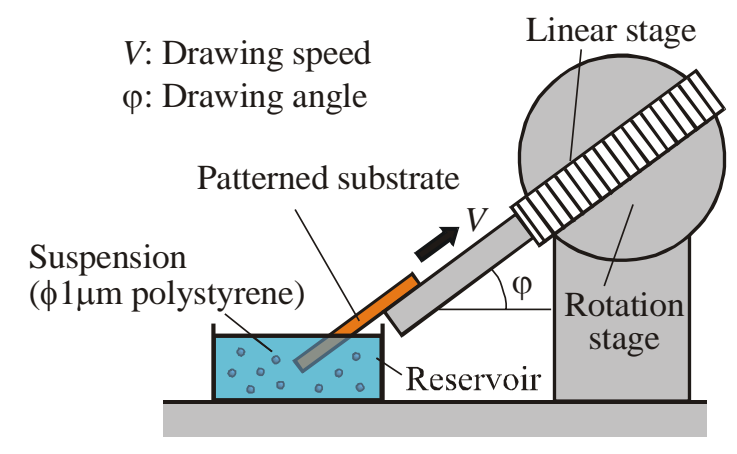

Figure 4: Experimental setup.

\subsection{Results and discussion}

The patterned substrate was drawn up from the suspension at speed $V=4 \mu \mathrm{m} / \mathrm{s}$ and angle $\varphi=30$. The geometry of hydrophilic/hydrophobic pattern used line and space at $W_{\text {OTS }}=50 \mu \mathrm{m}$ and $W_{\text {SiO2 }}=50 \mu \mathrm{m}$.

Figure 5 shows the scanning electron microscope (SEM) images of self-assembled particles on the substrate. The patterning field size was $1 \mathrm{~mm} \times 1 \mathrm{~mm}$ and smaller part within it was observed. The particles were assembled only on the $\mathrm{SiO}_{2}$ region, and they did not remain on the OTS region. Assembled particles were hexagonally packed in monolayer. Thus, this result is satisfactory though some defects just like cracks can be seen. The difference of the brightness on the substrate shows the difference of the surface material. It was found that the width of the assembled region was smaller than that of 
$\mathrm{SiO}_{2}$ region $50 \mu \mathrm{m}$. This is because the local spread on the $\mathrm{SiO}_{2}$ region took complicated shape and the final width of assemble is always smaller than that of the pattern. If an accurate size is required, the pattern width should be larger to compensate this effect.

The problem of the EB-lithography process is only the pattern size. As described before, EB-lithography requires long time. Therefore, particles cannot assemble large-scale region.

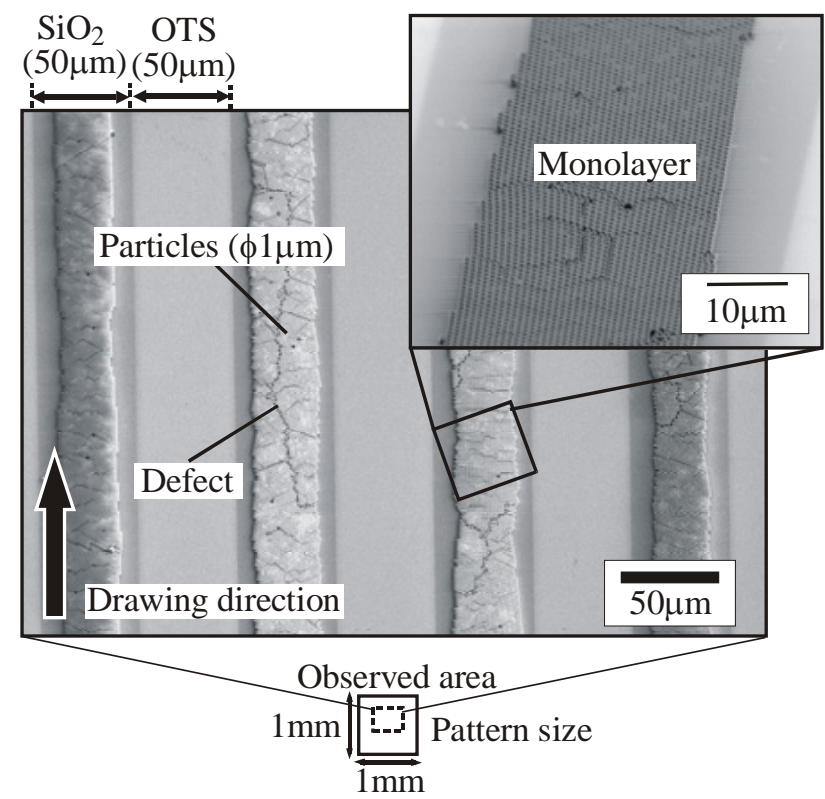

Figure 5: Self-assembled particles on patterned substrate fabricated by EB-lithography method $\left(W_{\text {SiO2 }}=50 \mu \mathrm{m}, W_{\text {OTS }}=50 \mu \mathrm{m}\right)$.

\section{Scale extension of the assembly using contact printing}

\subsection{Preparation of the substrates}

Contact printing has been applied for various material transfers and their scale extension [15-21]. Fabrication of hydrophilic/hydrophobic pattern with contact printing can, of course, extend its scale. However, basic properties have not been made clear. Thus, the printing process itself should be discussed before the self-assembly.

Figure 6 shows the process steps of fabricating OTS and $\mathrm{SiO}_{2}$ patterned substrate by contact printing. Firstly, the master mold with proper micro-structure was prepared with photolithography. Then, polydimethylsiloxane (PDMS) with $10 \mathrm{wt} \%$ of curing agent was cast onto the mold, cured at the temperature of 70 degrees for $2 \mathrm{~h}$, then, the PDMS was peeled off from the mold. The PDMS casting was inked with OTS. Finally, the inked OTS was printed on the $\mathrm{SiO}_{2}$ substrate. A contact pressure was applied during a period of time of which effect is discussed later. The pressure was kept at constant applying only the own weight of the PDMS casting.

The substrate was covered with $\mathrm{SiO}_{2}$ layer formed by soaking in $\mathrm{H}_{2} \mathrm{SO}_{4}: \mathrm{H}_{2} \mathrm{O}_{2}=3: 1$ solution for $2 \mathrm{~h}$ at temperature of 70 degrees. Just same with EB-lithography,
OTS reacts quickly with hydroxyl groups. Contact printing was carried out in a glove box purged with $\mathrm{N}_{2}$ gas.

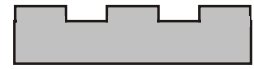

(1) Mold preparation

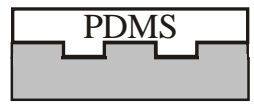

(2) PDMS casting

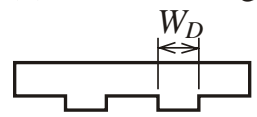

(3) Peel-off

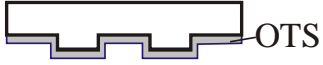

(4) Inking OTS

Contact time $t_{c}$

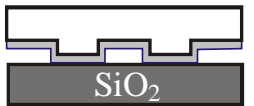

(5) Printing OTS

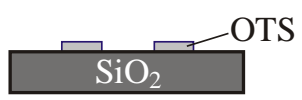

(6) Patterned OTS
Figure 6: Process step for OTS contact print.

It was found that the contact time in the printing process affect the properties of transferred OTS, though the OTS reacts with $\mathrm{SiO}_{2}$ in a short time theoretically. Figure 7 shows the effect of contact time on the contact angle of OTS region $\theta_{\text {oTs }}$ and pattern width of OTS Wots (See Fig.3). With the increase in contact time $t_{c}$, both of $\theta_{\text {oTs }}$ and Wотs became larger. It is considered that the contact time determined the number of transferred OTS molecules and the increase of WотS was caused by the elastic deformation of the mold. Thus, the contact time should be determined to obtain the desired mold shape $\left(W_{D}\right)$ should be designed to compensate the expected deformation to obtain desired WOTS based on these preliminary experiments.

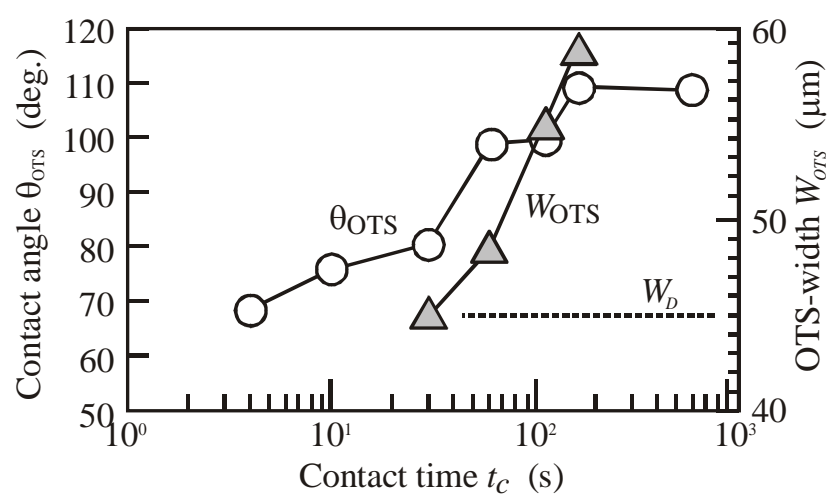

Figure 7: Effect of contact time $t_{C}$ on the contact angle $\theta_{\text {OTS }}$ and pattern width $W_{\text {OTS }}$.

Figure 8 shows the change in the contact angle after the contact print. The contact angle on OTS region is stable and no change is observed along time. However, the contact angle on $\mathrm{SiO}_{2}$ region increased with time up to 60 degree after 1000 hours. The droplet shapes are also shown in the figure. The effect of this change will not be neglected because the difference between the hydrophobic and hydrophilic property causes the location selective self-assemble discussed in this paper. From this results, it was found that the assemble process should be completed 
before 24 hours after the contact print. This condition is not so critical because it does not take long time for the assembling process.

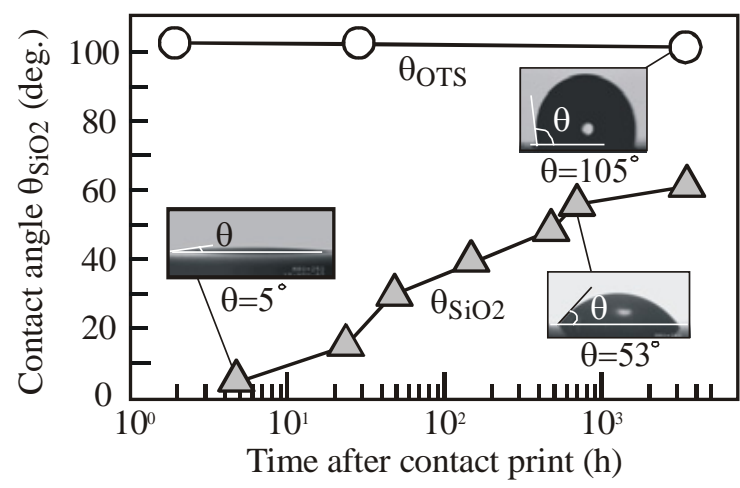

Figure 8: Change in the contact angle along time.

\subsection{Assembly results and discussion}

The patterned substrate was draw up from the suspension at drawing speed $V=8 \mu \mathrm{m} / \mathrm{s}$ and angle $\varphi=30$. The setup and suspension is same with the former experiments. The design of hydrophilic/hydrophobic pattern was line-and-space with Wотs $45 \mu \mathrm{m}$ and $W_{\text {SiO2 }} 50$ $\mu \mathrm{m}$. The contact angle of each region was 98 degrees for OTS and 5 degrees for $\mathrm{SiO}_{2}$ region respectively. The contact angle of OTS layer is smaller than that of the former experiments. The reason might be the imperfect structure of OTS. In the former experiments, the OTS has perfect monolayer based on the observation with AFM as shown in Fig.3.

It was found that the particles do not assemble on the substrate in one time draw-up and iterated draw-up was necessary to improve the coverage. Figure 9 shows the effect of drawing times on the coverage. The coverage $\varepsilon$ is defined as $P_{2} / P_{1}$, where $P_{2}$ is the covered area with particles on the $\mathrm{SiO}_{2}$ and $P_{1}$ is the area of $\mathrm{SiO}_{2}$ region. The maximum $\varepsilon$ is limited up to $85 \%$ because opening area remained between the particles. As shown in the figure, the coverage at the first drawing is just $10 \%$. However, after 15 times drawing, the coverage became larger than $70 \%$. Considering this coverage, the efficiency has

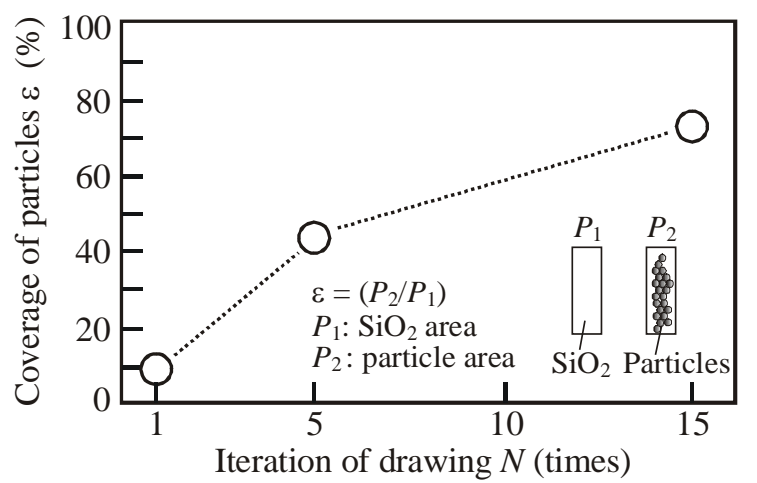

Figure 9: The effect of drawing time on coverage of particles. become lower than the former experiments. The difference of drawing speed might affect this change.

Figure 10 shows the observation results with laser microscope and SEM images of self-assembled particles after one time drawing. Hatching lines are superimposed on the image to indicate the location of $\mathrm{SiO}_{2}$ regions. The result is rather different from the intention, because the particles did not assemble on most of the $\mathrm{SiO}_{2}$ regions, where the particles should selectively assemble. However, particles assembled on just two regions on these patterns.

In addition, the layer is not monolayer but multilayered as shown in the magnified view in right upper of the figure. Many defects, line defects just like cracks or point defects just like vacancies, are observed in the layered region. Thus, the assembly was imperfect from the various viewpoints.

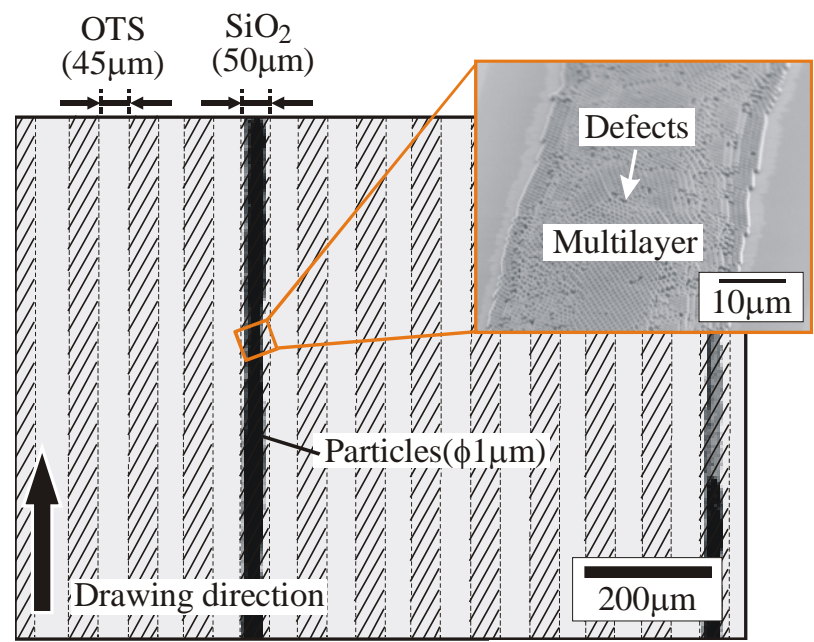

Figure 10: Assembled result after first drawing.

Figure 11 shows the observation results with laser microscope and SEM images that show the self-assembled particles on large-scale observation $(6 \mathrm{~mm} \times 4 \mathrm{~mm})$ on the assembled area of $10 \mathrm{~mm} \times 10 \mathrm{~mm}$. The dark stripe pattern denotes the assembled particles as shown in the magnified image in the upper left of the figure. However, the darkness or brightness of the stripe pattern varies with vertical position in the figure. This fluctuation might be the result of the fluctuation in drawing conditions such as speed during the process. In almost bright region, the particles assembled in monolayer as shown in Fig. 11 (a), while particles assembled in multilayer in dark region as shown in Fig. 11 (b). It was found that repetition of drawing, 15 times in this case, has improved the result because the assembled particles have good structure with less defects.

In some place in dark region, the particles assembled also on OTS region as shown in Fig. 11 (c). The reason is considered as the imperfect structure of the OTS layer as described above though it is not confirmed. The contact angle on OTS region was small and almost at the boundary between hydrophobic and hydrophilic characteristic, the contact angle of 90 degrees. The 

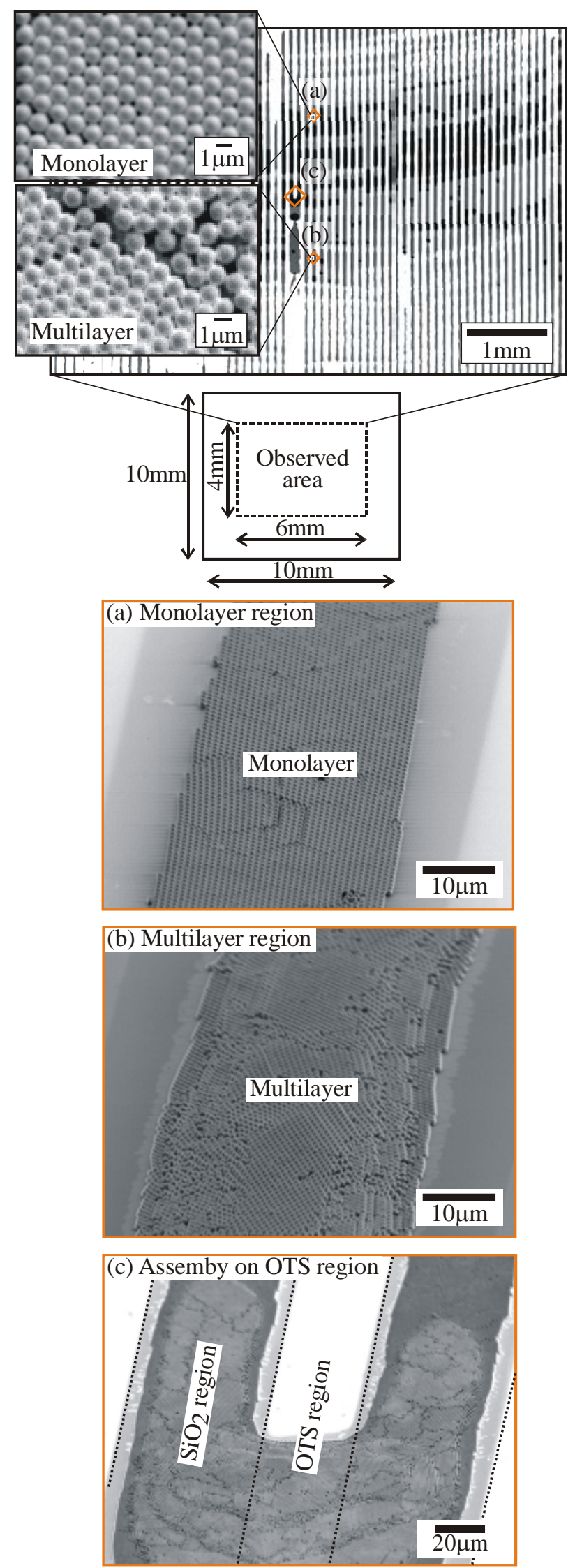

Figure 11: Assembled results after 15 times iteration on large-scale patterned substrate fabricated by contact printing method

$\left(\mathrm{W}_{\mathrm{SiO} 2}=50 \mu \mathrm{m}, \mathrm{W}_{\mathrm{OTS}}=45 \mu \mathrm{m}\right)$. contrast of wettability should be made as high as possible, that is, the contact angle should be large on hydrophobic region and it should be small on hydrophilic region to obtain good assembly.

\section{Conclusion}

This paper aimed to demonstrate the self-assembly of fine particles by dip coating to hydrophilic/hydrophobic patterned substrates. The results as summarized as follows:

- Using electron-beam lithography, self-assembled packed-structure in monolayer can be obtained selectively on the hydrophilic region for water-based suspension.

- Contact printing process can scale up the size of assembly, $10 \mathrm{~mm} \times 10 \mathrm{~mm}$ in this study, though much attention should be paid for printing conditions.

\section{Reference}

[1] Hirai, T., Hayashi, S., 1999, Lens functions of polymer microparticle arrays, Colloids and Surfaces A, Vol.153, pp.503-513.

[2] Yang, H., Zhu, Y., 2007, Glucose biosensor based on nano-SiO2 and "unprotected" $\mathrm{Pt}$ nanoclusters, Biosensors and Bioelectronics, Vol.22, pp.2989-2993.

[3] Xua, J., Zhang, Y., Lia, G., Zhu, J., 2004, An electrochemical biosensor constructed by nanosized silver particles doped sol-gel film, Materials Science and Engineering C, Vol.24, pp.833-836.

[4] Nakanishi, T., Hiraoka, T., Fujimoto, A., Saito, S., Asakawa, K., 2006, Nano-patterning using an embedded particle monolayer as an etch mask, Microelectronic Engineering, Vol.83, pp.1503-1508.

[5] Astratov, V. N., et. al., 1996, Photonic band gaps in 3D ordered fcc silica matrices, Phys. Lett . A, Vol.222, pp.349-353.

[6] Pan, G., Kesavamoorthy, R., and Asher, S. A., 1997, Optically Nonlinear Bragg Diffracting Nanosecond Optical Switches, Phys. Rev. Lett., Vol.78, pp.3860-3863.

[7] Asher, S. A., Weissman, J. M., Tikhonov, A., Coalson, R. D., and Kesavamoorthy, R., 2004, Diffraction in crystalline colloidal-array photonic crystals, Phys. Rev. E, Vol.69, pp.066619-1-066619-14

[8] Meseguer, F., 2005, Colloidal crystals as photonic crystals, Colloids and Surfaces A, Vol.270-271, pp.1-7

[9] Cojoc, D., Garbin, V., Ferrari, E., Businaro, L., Romanato, F., Fabrizio, E., 2005, Laser trapping and micro-manipulation using optical vortices, Microelectronic Engineering, Vol.78-79, pp.125-131.

[10] Schnelle, T., Muller, T., Hagedorn, R., Voigt, A., Fuhr, G., 1999, Single micro electrode-dielectro phoretic tweezers for manipulation ofsuspended cells and particles, Biochimica et Biophysica Acta, Vol.1428, pp.99-105.

[11] Dimitrov, S. A., and Nagayama, K., 1996, Continuous Convective Assembling of Fine Particles into Two-Dimensional Arrays on Solid Surfaces, Langmuir, 
Vol.12, pp.1303-1311.

[12] Matsushita, S. I., Miwa, T., Tryk, D. A., and Fujishima, A., 1998, New mesostructured porous TiO2 surface prepared using a two-dimensional array-based template of silica particles, Langmuir, Vol.14, pp.6441-6447.

[13] Masuda, Y., Itoh, M., Yonezawa, T., and Koumoto, K., 2002, Low-dimensional arrangement of $\mathrm{SiO} 2$ particles, Langmuir, Vol.18, pp.4155-4159.

[14] Kaneko, A., Moronuki, N. Shibata, T., Kogiso, J., Uchida, K., and Kubo, T., 2006, An Application of Dip -coating Technique to Fabrication of Self-assembled Microstructure on Hydrophilic/Hydrophobic-patterned Substrate, Proc. of euspen, Vol.2, pp.288-291.

[15] van Delft, F. C. M. J. M., van den Heuvel, F. C., Kuiper, A. E. T., Thune, P. C., and Niemantsverdriet, J.W., 2004, Micro-contact printing on oxide surfaces for model catalysts using e-beam written masters in hydrogen silsesquioxana, Microelectronic Eng., Vol.73-74, pp.202-208

[16] Kumar, A., Biebuyck, H. A., and Whiteside, G. M., 1994, Patterning Self-Assembled Monolayers: Apprications in Materials science, Langmuir, Vol.10, pp.1498-1511

[17] Heule, M., Schonholzer, U. P., Gauckler, L., J., 2004, Patterning colloidal suspensions by selective wetting of microcontact-printed surfaces, Journal of the European Ceramic Society, Vol.24, pp.2733-2739

[18] John, P., M., S., Craighead, H., G., 1995, Microcontact printing and pattern transfer using trichlorosilanes on oxide substrates, Appl. Phys. Lett., Vol.68, pp.1022-1024

[19] Jeon, N., L., Clem, P., Jung, D., Y., Lin, W., Girolami, G., S., Payne, D., A., and Nuzzo, R., G., 1997, Additive Fabrication of Integrated Ferroelectric Thin-Film Capacitors Using Self-Assembled Organic Thin-Film Template, Adv. Mater., Vol.9, pp.891-895

[20] Wang, D., Thomas, S., G., Wang, K., L., Xia, Y., Whiteside, G. M., 1997, Nanometer scale patterning and pattern transfer on amorphous $\mathrm{Si}$, crystalline $\mathrm{Si}$, and $\mathrm{SiO} 2$ surfaces using self-assembled monolayers, Appl. Phys. Lett., Vol.70, pp.1593-1595

[21] Payne, D., A., Clem, P., G., 1999, Monolayer-Mediated Patterning of Integrated Electroceramics, Journal of Electroceramics 3, Vol.2, pp.163-172. 